\title{
Effects of Flavonoids on Xanthine Oxidation as well as on Cytochrome $c$ Reduction by Milk Xanthine Oxidase
}

\author{
Masayoshi IIO, Yoshimi ONO, Setsumi KaI, \\ and Michi Fukumoto ${ }^{1}$ \\ Laboratory of Nutritional Biochemistry, Department of Food and \\ Nutrition, Faculty of Living Science, Kumamoto Women's \\ University, Kumamoto 862, Japan
}

(Received July 25, 1986)

\begin{abstract}
Summary That flavonoids inhibit xanthine oxidase from cow milk was confirmed by measuring oxygen consumption with an oxygen electrode. In contrast, flavonoids did not inhibit glucose oxidase, another oxygen consuming enzyme. Among the flavonoids tested, quercetin, kaempferol, myricetin, chrysin, quercitrin, and morin were potent inhibitors of xanthine oxidase; their inhibition rates $(\%)$ were $80,70,69,62,59$, and 51 at $100 \mu \mathrm{M}$ (except chrysin at $50 \mu \mathrm{M}$ ), respectively. The xanthine oxidaseinhibiting activities of the flavonoids were not always well correlated with the suppressive activities of the flavonoids on cytochrome $c$ reduction by a xanthine-xanthine oxidase system. The inhibition of xanthine oxidase by quercetin was not affected by cupric ion. The partition rates of the flavonoids between $n$-butanol and a buffer solution seemed to account for some of the inhibition.
\end{abstract}

Key Words flavonoids, xanthine oxidase, xanthine oxidation, cytochrome $c$ reduction

The xanthine-xanthine oxidase system [EC 1.2.3.2] generates uricate and superoxide, both of which have very important physiological roles. Uricate is useful as an antioxidative agent present in the body, although it is also the cause of gout. Superoxide causes damage to biomolecules such as nucleic acids, proteins, and lipids, while it is useful in degrading foreign matter within lymphocytes. We reported on the inhibitory activities of flavonoids on the xanthine oxidase reaction in one physiological and biochemical study of flavonoids (1-4). In the previous study (5), the reactions were followed by measuring uricate formation at $295 \mathrm{~nm}$, which is in the absorbing region of the flavonoids present in the reaction system. This prompted us to adopt another measurement method using a different principle which could not be interfered with by flavonoid absorption. We also

1 飯尾雅嘉, 小野よしみ, 甲斐節美, 福元みち 
wanted to know if flavonoids inhibit another type of oxygen-consuming enzymes such as glucose oxidase [EC 1.1.3.4], and whether or not cupric ion suppresses the inhibitory activities of flavonoids as reported by other investigators. Furthermore we tried to estimate physical properties of flavonoids, such as partition rates, in order to check their correlations with the inhibitory activities.

\section{EXPERIMENTAL}

Chemicals. Xanthine oxidase from milk and glucose oxidase from Aspergillus niger were obtained from Boehringer. The sources of the flavonoids tested have been described elsewhere. Some of the flavonoids were gifts from Prof. T. Nakabayashi of Shizuoka University, or from Dr. T. Yamagishi of Hokkaido Institute of Public Health. Other reagents were obtained from commercial sources and were used without further purification.

Xanthine oxidase activity. 1) Oxygen electrode method. The reaction mixture contained $1.38 \mathrm{ml}$ of $50 \mathrm{~mm}$ potassium phosphate buffer, $\mathrm{pH} 7.8,0.180 \mathrm{ml}$ of $1 \mathrm{~mm}$ disodium ethylenediaminetetraacetate, $0.018 \mathrm{ml}$ of $10 \mathrm{~mm}$ xanthine, $0.180 \mathrm{ml}$ of $10 \mathrm{~mm}$ flavonoid dissolved in dimethyl sulfoxide, and $0.050 \mathrm{ml}$ of the enzyme solution diluted appropriately. The reaction was done at $30^{\circ} \mathrm{C}$ in a temperaturecontrolled cell equipped with an oxygen electrode connected to a recorder through a DO converter (Iijima Seimitsu Kogyo). The buffer solution was saturated with oxygen by bubbling with air before use. The reaction rate was determined by measuring the voltage change for $3 \mathrm{~min}$. 2) UV absorption method. The reaction mixture contained $1.56 \mathrm{ml}$ of $50 \mathrm{~mm}$ potassium phosphate buffer, $\mathrm{pH} 7.8,0.20 \mathrm{ml}$ of $1 \mathrm{~mm}$ disodium ethylenediaminetetraacetate, $0.20 \mathrm{ml}$ of $10 \mathrm{~mm}$ xanthine, $0.02 \mathrm{ml}$ of $10 \mathrm{~mm}$ flavonoid dissolved in dimethyl sulfoxide, $0.04 \mathrm{ml}$ of $2.5 \mathrm{~mm}$ cupric sulfate, and $0.04 \mathrm{ml}$ of the enzyme solution diluted appropriately. The reaction was done at $30^{\circ} \mathrm{C}$ in a cuvette in a temperature-controlled cuvette holder attached to a UV-240 Shimadzu spectrophotometer. The absorbance at $295 \mathrm{~nm}$ was measured during the initial $60 \mathrm{sec}$.

Cytochrome c reduction by xanthine-xanthine oxidase system. The reaction mixture contained $0.68 \mathrm{ml}$ of $50 \mathrm{~mm}$ sodium phosphate buffer, $\mathrm{pH} 7.8,0.10 \mathrm{ml}$ of $1 \mathrm{~mm}$ disodium ethylenediaminetetraacetate, $0.10 \mathrm{ml}$ of $10 \mathrm{~mm}$ xanthine, $0.10 \mathrm{ml}$ of $100 \mu \mathrm{M}$ cytochrome $c, 0.01 \mathrm{ml}$ of $10 \mathrm{~mm}$ flavonoid dissolved in dimethyl sulfoxide, and $0.02 \mathrm{ml}$ of xanthine oxidase solution. The reaction was done at $30^{\circ} \mathrm{C}$ in a cuvette as in the xanthine oxidase activity measurement by absorption change. The absorbance at $550 \mathrm{~nm}$ was followed for $1 \mathrm{~min}$ after the enzyme addition.

Glucose oxidase activity. The reaction mixture contained $1.80 \mathrm{ml}$ of $100 \mathrm{~mm}$ glucose dissolved in $50 \mathrm{~mm}$ sodium phosphate buffer, $\mathrm{pH} 6.5,0.010 \mathrm{ml}$ of $10 \mathrm{~mm}$ flavonoid in dimethyl sulfoxide, $0.025 \mathrm{ml}$ of diluted catalase $(180 \mu \mathrm{g})$, and $0.050 \mathrm{ml}$ of glucose oxidase solution $(0.010 \mathrm{mg} / \mathrm{ml})$. The reaction was done at $37^{\circ} \mathrm{C}$ in a manner similar to the xanthine oxidase activity measurement.

Determination of partition rates. One 200 th volume of a $10 \mathrm{~mm}$ flavonoid 
solution $(0.025 \mathrm{ml})$ was added to the mixture of $2.50 \mathrm{ml}$ of sodium phosphate buffer solution, $\mathrm{pH} 6.8$, and $2.50 \mathrm{ml}$ of either $n$-butanol or ethyl acetate. The total mixture was stirred well and left to stand. The two separate layers were taken and the absorption spectra were measured separately. The partition rates were calculated from the absorbances at the absorption maxima of the longest wavelength; the absorbance of the aqueous layer was divided by that of the organic solvent layer.

\section{RESULTS}

Flavonoid-induced inhibition of xanthine oxidase activity measured by oxygen electrode method

Flavonoids were shown to inhibit the xanthine oxidase reaction as can be seen in Table 1. Quercetin, kaempferol, myricetin, chrysin, quercitrin, and morin were very inhibitory. Fisetin, gossypitrin, baicalein, naringenin, herbacitrin, and wogonin glucuronide were moderately inhibitory, while rutin, myricitrin, robinin, baicalin, 3hydroxyflavone, hesperidin, flavone, naringin, and apiin were weak to inactive inhibitors (data not shown). Figure 1 shows the correlation of inhibition rates measured by the absorption method in the previous study (5) with those measured by the oxygen electrode method in the present study. The rates determined by both methods correlated well with each other.

Effect of flavonoids on cytochrome c reduction by xanthine-xanthine oxidase system

Table 2 shows that some flavonoids clearly suppressed or even reversed the absorbance increase at $550 \mathrm{~nm}$ which is considered to reflect the cytochrome $c$ reduction caused by the xanthine-xanthine oxidase system. However, the suppressive activity of each flavonoid was not always parallel to that seen in its

Table 1. Inhibition of xanthine oxidase by flavonoids as measured by oxygen consumption.

The reaction was followed for the initial $3 \mathrm{~min}$ with an oxygen electrode connected to a recorder through a DO converter. The reaction was done in a temperature-controlled cell $\left(30^{\circ} \mathrm{C}\right)$. The values described are the average of triplicate measurements. The final concentration of each flavonoid was $100 \mu \mathrm{M}$, except that of chrysin was $50 \mu \mathrm{m}$ because of its low solubility. Experimental details are described in the text.

\begin{tabular}{lc}
\hline Flavonoid & Inhibition rate $(\%)$ \\
\hline Quercetin & 80 \\
Kaempferol & 70 \\
Myricetin & 69 \\
Chrysin & 62 \\
Quercitrin & 59 \\
Morin & 51 \\
\hline
\end{tabular}

Vol. 32, No. 6, 1986 


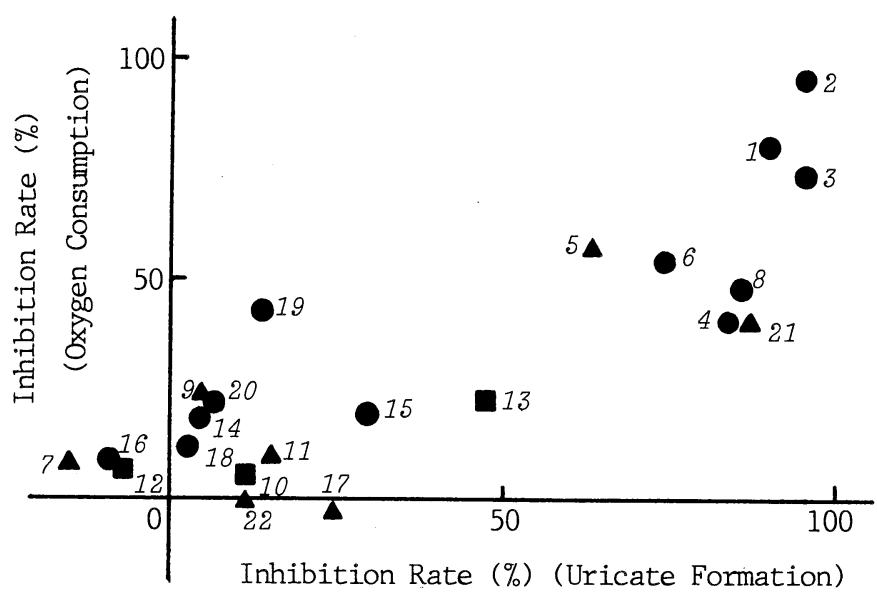

Fig. 1. Comparison of xanthine oxidase inhibition measured by the oxygen consumption and absorption methods. The values in the ordinate were taken from Table 1 and those in the abscissa were taken from a previous study (5). Flavonol

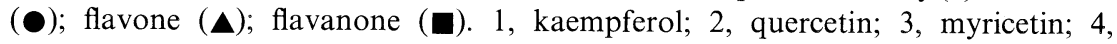
quercitrin; 5, chrysin; 6, morin; 7, flavone; 8, fisetin; 9, wogonin glucuronide; 10, naringin; 11 , baicalin; 12 , hesperidin; 13 , naringenin; 14 , rutin; 15 , myricitrin; 16, 3hydroxyflavone; 17, apiin; 18, robinin; 19, gossypitrin; 20, herbacitrin; 21 , baicalein; 22 , wogonin.

xanthine oxidase inhibition as measured by the oxygen electrode method. For example, rutin was not a potent inhibitor of xanthine oxidase, although it suppressed the cytochrome $c$ reduction very strongly.

\section{Effect of flavonoids on glucose oxidase activity}

Some flavonoids were added to the glucose oxidase system to determine whether they were also inhibitory to oxygen consuming enzymes other than xanthine oxidase. As can be seen in Table 3, they were not porent inhibitors of glucose oxidase, in contrast to their action on xanthine oxidase.

\section{Effect of cupric ion on xanthine oxidase inhibition by quercetin}

In order to determine whether flavonoids such as quercetin inhibit xanthine oxidase by binding with metal contained in the enzyme, cupric ion was added to the reaction mixture. Cupric ion is often used to elucidate the mechanism of biochemical effect of flavonoids. Table 4 shows the results. Cupric ion did not show any significant effect on xanthine oxidase inhibition by quercetin.

\section{Partition rates of flavonoids and their inhibitory activities}

The partition rates of flavonoids between an aqueous buffer solution and an organic solvent were measured to clarify the possible mechanism of xanthine 
Table 2. Effect of flavonoids on cytochrome $c$ reduction caused by xanthine-xanthine oxidase system.

The reaction was followed for the initial $1 \mathrm{~min}$ by absorbance change at $550 \mathrm{~nm}$ due to cytochrome $c$ reduction. The reaction was done in a temperature-controlled cuvette $\left(30^{\circ} \mathrm{C}\right)$. The final concentration of each flavonoid was $100 \mu \mathrm{m}$, except that those of chrysin, 3-hydroxyflavone, and flavone were 50,10 , and $10 \mu \mathrm{M}$, respectively, because of their low solubility. Experimental details are described in the text. The relative absorbance increase $(\%)$ is expressed as (absorbance increase in the test)/(absorbance increase in the control) $\times 100$. The control contained DMSO instead of flavonoid. The values described are the average of triplicate measurements.

\begin{tabular}{lclc}
\hline Flavonoid & $\begin{array}{c}\text { Relative } \\
\text { absorbance } \\
\text { increase (\%) }\end{array}$ & Flavonoid & $\begin{array}{c}\text { Relative } \\
\text { absorbance } \\
\text { increase }(\%)\end{array}$ \\
\hline Morin & -38 & Baicalin & 13 \\
Rutin & -31 & Robinin & 71 \\
Kaempferol & -11 & Wogonin glucuronide & 77 \\
Fisetin & -6 & Naringenin & 84 \\
Myricitrin & -5 & Chrysin & 88 \\
Quercitrin & -4 & Naringin & 88 \\
Gossypitrin & -3 & Wogonin & 90 \\
Herbacitrin & -3 & Hesperidin & 93 \\
Baicalein & -3 & 3-Hydroxyflavone & 100 \\
Quercetin & -1 & Flavone & 100 \\
Myricetin & 1 & Apiin & 100 \\
\hline
\end{tabular}

Table 3. Effect of flavonoids on glucose oxidase activity as measured by oxygen consumption.

The reaction was followed with an oxygen electrode connected to a recorder through DO converter. The reaction was done in a temperature-controlled cell $\left(37^{\circ} \mathrm{C}\right)$. The final concentration of each flavonoid was $100 \mu \mathrm{M}$. Experimental details are described in the text. The values described are the average of triplicate measurements.

\begin{tabular}{lclc}
\hline Flavonoid & $\begin{array}{c}\text { Inhibition } \\
\text { rate }(\%)\end{array}$ & Flavonoid & $\begin{array}{c}\text { Inhibition } \\
\text { rate }(\%)\end{array}$ \\
\hline Rutin & 24 & Kaempferol & 11 \\
Myricetin & 18 & Herbacitrin & 11 \\
Myricitrin & 16 & Gossypitrin & 10 \\
Quercitrin & 15 & Robinin & 9 \\
Quercetin & 13 & & \\
\hline
\end{tabular}

oxidase inhibition by flavonoids. It can be said that the more a flavonoid is soluble in $n$-butanol, the more potent an inhibitor it is, with some exceptions such as flavone and 3-hydroxyflavone (Fig. 2). The partition rates between ethyl acetate and an 
Table 4. Effect of cupric ion on quercetin-induced inhibition of xanthine oxidase. The reaction was done in a temperature-controlled cuvette $\left(37^{\circ} \mathrm{C}\right)$ and was followed by absorption change either in the absence or in the presence of cupric ion. The final concentrations of quercetin and cupric ion were 100 and $50 \mu \mathrm{M}$, respectively. The values described are the average of triplicate measurements. Experimental details are described in the text.

\begin{tabular}{cc}
\hline Cupric ion & Inhibition rate $(\%)$ \\
\hline+ & 81 \\
- & 84 \\
\hline
\end{tabular}

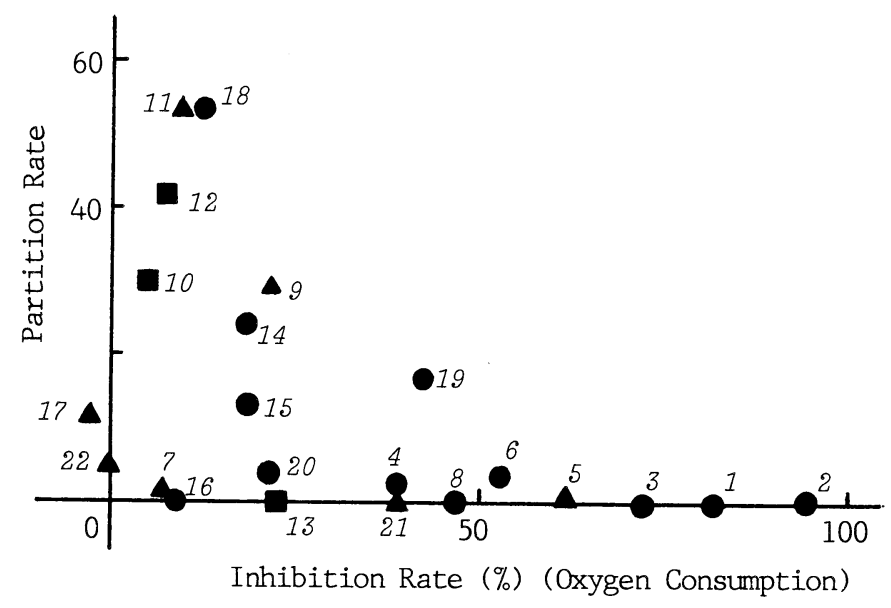

Fig. 2. Correlation of partition rates with xanthine oxidase inhibition rates of flavonoids. The partition rates of the flavonoids between $n$-butanol and an aqueous buffer solution were calculated from the absorbances of each layer after partitioning. Experimental details are described in the text. The symbols and numbers for the flavonoids are the same as in the legend to Fig. 1.

aqueous buffer did not show similar results (data not shown).

\section{DISCUSSION}

An oxygen electrode is a very convenient tool to measure oxygen dissolved in aqueous media, and can be used in enzymatic reactions consuming oxygen regardless of the coloration or absorption of the reaction mixtures. Thus we adopted this tool to estimate the inhibitory activities of flavonoids on xanthine oxidase as well as on glucose oxidase. Some of the flavonoids tested were shown to be inhibitors of xanthine oxidase. The data described in the previous study were 
thus confirmed by this method. Hence the spectrophotometric method was considered to be useful in xanthine oxidase activity measurement even in the presence of flavonoids. On the other hand, they were not inhibitors of glucose oxidase, another oxygen consuming enzyme. We do not know for the time being why this difference occurs. Quercetin, kaempferol, myricetin, chrysin, quercitrin, and morin, most of which are flavonols, were potent inhibitors of xanthine oxidase. Glycosidation of the aglycons appeared to decrease their inhibitory activities.

Cytochrome $c$ is reduced by superoxide generated by the xanthine-xanthine oxidase system. The reaction can be followed by the absorption increase at $550 \mathrm{~nm}$ due to an increase in the reduced form of cytochrome $c$. Xanthine oxidase inhibition by a certain factor was expected to result in lowered absorption increase. Thus we added flavonoids to the reaction mixture of cytochrome $c$ reduction to ascertain the inhibitory activities of the flavonoids. Those flavonoids like quercetin that inhibited the xanthine oxidase reaction did suppress the cytochrome $c$ reduction. However, such suppression was also observed with some flavonoids that were not inhibitory to xanthine oxidase. Rutin was such an example. Rutin and some other glycosidic flavonoids suppressed the reduction by a mechanism other than xanthine oxidase inhibition. Almost all the reduction suppressing flavonoids were flavonols with more than 4 hydroxyl groups or their glycosides. The only exceptions in this study were baicalein and its glucuronide, baicalin that were classified as flavones. Flavanones such as naringenin, naringin, and hesperidin were not active. Simply having a glycosidic moiety did not seem to be a sufficient condition for the reduction suppressing activity as can be seen in the case of robinin, naringin, or apiin.

Schwartz et al.(6) reported that divalent cations, especially cupric ion, suppressed the cytological activity of some flavonoids. However, cupric ion did not affect the xanthine oxidase inhibition by quercetin in our study. The absorption spectrum of quercetin was not affected by the presence of cupric ion, indicating that the cation did not interact with the flavonoid (data not shown).

Flavonoids have been reported to inhibit a variety of enzymes such as glyoxalase I(1), $\alpha$-glucosidase (2), and dextran sucrase(3) as well as xanthine oxidase described here. In addition, they inhibit other enzymes, as reported in the literature (7). But unlike phenol or some other compounds, they are not non-specific inhibitors of the enzymes; they did not inhibit yeast $\beta$-fructosidase (2), a certain type of galactosidase (4), or trypsin (unpublished data). Among flavonoids inhibitory to xanthine oxidase, there were various grades of activity from very potent to weak. We wanted to know what made flavonoids act differently. We expected that certain physico-chemical properties could explain this. We examined the partition rates of the flavonoids between an organic solvent and an aqueous buffer solution in addition to their surface activities. The partition rates between $n$-butanol and an aqueous buffer solution appeared to explain some part of the inhibitory activities of the flavonoids to xanthine oxidase, while a similar result was not obtained from the partition between an aqueous buffer solution and ethyl acetate which is often used to extract flavonoids from plants. Apparently solubility in an organic solvent such 
as $n$-butanol is necessary to some extent for a certain flavonoid to have maximal inhibitory activity; if it is too hydrophilic (robinin or rutin) or too hydrophobic (flavone and so forth), it cannot attach to an appropriate site on the enzyme. The number and site of phenolic hydroxyl groups might have some significance in this respect. The surface activities measured by a surface tensometer did not seem to be related to the inhibitory activities (data not shown). Thus the structure-activity relationship still remains to be elucidated.

We express our thanks to Prof. T. Nakabayashi and Dr. T. Yamagishi for their generous gifts of certain flavonoids.

\section{REFERENCES}

1) Iio, M., Himeno, S., Miyauchi, K., Mikumo, K., and Ohta, N. (1983): Effect of flavonoids on glyoxalase I activity. Nippon Nogei Kagaku Kaishi (J. Agric. Chem. Soc. Jpn.), 57, 765-769; Iio, M., Ishimoto, S., Nishida, Y., Shiramizu, T., and Yunoki, H. (1986): Effects of baicalein, a flavonoid, and other antiinflammatory agents on glyoxalase-I activity. Agric. Biol. Chem., 50, 1073-1074.

2) Iio, M., Yoshioka, A., Imayoshi, Y., Koriyama, C., and Moriyama, A. (1984): Effect of flavonoids on $\alpha$-glucosidase and $\beta$-fructosidase from yeast. Agric. Biol. Chem., 48, $1559-1563$.

3) Iio, M., Uyeda, M., Iwanami, T., and Nakagawa, Y. (1984): Flavonoids as a possible preventive of dental caries. Agric. Biol. Chem., 48, 2143-2145.

4) Iio, M. (1986): Purification of sucrase from porcine intestinal mucosa membrane and effects of flavonoids on glycosidases including the sucrase. J. Kumamoto Women's Univ., 38, 60-69.

5) Iio, M., Moriyama, A., Matsumoto, Y., Takaki, N., and Fukumoto, M. (1985): Inhibition of xanthine oxidase of flavonoids. Agric. Biol. Chem., 49, 2173-2176.

6) Schwartz, A., and Middleton, E., Jr. (1984): Comparison of the effects of quercetin with those of other flavonoids on the generation and effector function of cytotoxic $\mathrm{L}$ lymphocytes. Immunopharmacology, 7, 115-126.

7) Havsteen, B. (1983): Flavonoids, a class of natural products of high pharmacological potency. Biochem. Pharmacol., 32, 1141-1148. 